\title{
Loss of HITS (FAM107B) expression in cancers of multiple organs: tissue microarray analysis
}

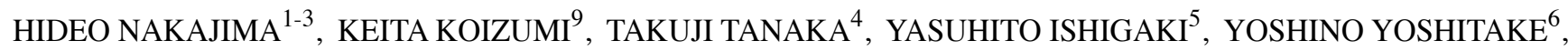 \\ HIDETO YONEKURA $^{6}$, TSUTOMU SAKUMA ${ }^{7}$, TOSHIHIRO FUKUSHIMA ${ }^{8}$, HISANORI UMEHARA ${ }^{8}$, \\ SOICHIRO UENO $^{1}$, TOSHINARI MINAMOTO ${ }^{10}$ and YOSHIHARU MOTOO $^{3}$ \\ ${ }^{1}$ Department of Oncology, Ageo Central General Hospital, Ageo, Saitama; ${ }^{2}$ Center for AIDS Research, \\ Kumamoto University, Honjo, Kumamoto; Departments of ${ }^{3}$ Medical Oncology, ${ }^{4}$ Pathology, ${ }^{5}$ Medical Research Institute, \\ Departments of ${ }^{6}$ Biochemistry, ${ }^{7}$ Thoracic Surgery and ${ }^{8}$ Hematology and Immunology, Kanazawa Medical University, \\ Uchinada, Ishikawa; ${ }^{9}$ Department of Biophysical Genetics, Graduate School of Medical Science; \\ ${ }^{10}$ Division of Translational and Clinical Oncology, Cancer Research Institute, \\ Kanazawa University, Kanazawa, Ishikawa, Japan
}

Received March 27, 2012; Accepted May 29, 2012

DOI: $10.3892 /$ ijo.2012.1550

\begin{abstract}
Family with sequence similarity 107 (FAM107) proteins consist of two subtypes, FAM107A and FAM107B in mammals, possessing a conserved N-terminal domain of unknown function. Recently we found that FAM107B, an $18 \mathrm{kDa}$ nuclear protein, is expressed in a broad range of tissues and is downregulated in gastrointestinal cancer. Because FAM107B expression is amplified by heat-shock stimulation, we designated it heat shock-inducible tumor small protein (HITS). Although data related to FAM107A as a candidate tumor suppressor have been accumulated, little biological information is available for HITS. In the present study, we examined HITS expression using immunohistochemistry with tissue microarrays and performed detailed statistical analyses. By screening a high-density multiple organ tumor and normal tissue microarray, HITS expression was decreased in tumor tissues of the breast, thyroid, testis and uterine cervix as well as the stomach and colon. Further analysis of tissue microarrays of individual
\end{abstract}

Correspondence to: Dr Hideo Nakajima, Department of Oncology, Ageo Central General Hospital, 1-10-10 Kashiwaza, Ageo, Saitama 362-8588, Japan

E-mail: nakajima.h@ach.or.jp

Abbreviations: FAM107, family with sequence similarity 107 ; HITS, heat-shock-inducible tumor small protein; HSP, heat-shock protein; TSG, tumor suppressor gene; DUF, domain of unknown function; HAT, histone acetyltransferase; ER, estrogen receptor; $\mathrm{PR}$, progesterone receptor; HER2, human epidermal growth factor receptor 2; IDC, invasive ductal carcinoma; DCIS, ductal carcinoma in situ; CIN, cervical intraepithelial neoplasia; HPV, human papilloma virus; PCNA, proliferating cell nuclear antigen; FNAC, fine needle aspiration cytology; FA, focal adhesion

Key words: family with sequence similarity 107B, heat-shock protein, tumor suppressor gene, tissue array organs showed that loss of HITS expression in cancer tissues was statistically significant and commonly observed in distinct organs in a histological type-specific manner. The HITS expression intensity was inversely correlated with the primary tumor size in breast and thyroid cancers. In addition, effects of tetracycline-inducible HITS expression on tumor growth were investigated in vivo. Forced expression of HITS inhibited tumor xenograft proliferation, compared with the mock-treated tumor xenograft model. These results show that loss of HITS expression is a common phenomenon observed in cancers of distinct organs and involved in tumor development and proliferation.

\section{Introduction}

Family with sequence similarity 107 (FAM107) proteins are conserved beyond species: they are found in mammals, Xenopus, fish and Drosophila. They are characterized by a common N-terminal domain of unknown function (DUF1151) with no homology match to other functional conserved domains. Mammals have two genes, FAM107A and FAM107B, respectively encoding the proteins of 144 amino acids (aa) and 131 aa (http://www.uniprot.org/uniprot). C-terminal variable regions of FAM107 carry a coiled-coil domain that has been identified in many nuclear proteins including transcription factors, suggesting a role of FAM107 in regulating gene transcription. Analysis of protein-protein interactions revealed that FAM107A and FAM107B interact, respectively, with transcriptional adaptor $2 \alpha$ and $3 \alpha$ (Tada2 $\alpha$ and Tada $3 \alpha$ ) (1-3). In fact, Tada $2 \alpha$ and Tada $3 \alpha$ are reciprocal binding partners and core proteins of the histone acetyltransferase (HAT) complex, suggesting that FAM107 family proteins can modulate the structure and function of HAT complexes that are involved in chromatin structure modification for gene transcription and acetylation of many proteins $(4,5)$.

Because of epigenetic silencing, FAM107A is downregulated or deleted from cancer of many types such as non-small cell lung, renal cell, and prostate cancers and astrocytoma (6-10). 
Table I. Formats of the tissue microarray.

\begin{tabular}{|c|c|c|}
\hline Tissue microarray ${ }^{\mathrm{a}}$ & Organ & Specificity \\
\hline MC5003 & Multiple & $\begin{array}{l}\text { High-density multiple organ tumor and normal tissue microarray, containing } 18 \\
\text { types of tumor ( } 20 \text { spots/type) and normal controls ( } 5 \text { spots/type) }\end{array}$ \\
\hline BRM961 & Breast & $\begin{array}{l}\text { Breast carcinoma metastatic tissue microarray, containing } 96 \text { spots of breast cancer, } \\
\text { with } 72 \text { matched metastatic breast cancer (in lymph nodes) and } 24 \text { normal tissue }\end{array}$ \\
\hline BR1503 & Breast & $\begin{array}{l}\text { Breast carcinoma tissue microarray (including TNM and pathology grade, with } \\
\text { IHC results of HER } 2, \mathrm{ER}, \mathrm{PR}, \mathrm{P} 53 \text { and Ki-67), containing } 6 \text { spots of normal tissue, } \\
6 \text { fibroadenoma, } 4 \text { cystosarcoma phyllodes, } 14 \text { intraductal carcinoma, } 120 \text { invasive } \\
\text { ductal carcinoma }\end{array}$ \\
\hline TH807 & Thyroid & $\begin{array}{l}\text { Thyroid disease spectrum tissue microarray, } 10 \text { spots of each types } \\
\text { (papillary carcinoma, follicular carcinoma, metastatic carcinoma, adenoma, } \\
\text { goiter, thyroiditis, cancer adjacent normal and normal tissue) }\end{array}$ \\
\hline CR602 & Uterine cervix & $\begin{array}{l}\text { Uterine cervical disease spectrum tissue microarray, containing } 30 \text { spots } \\
\text { of uterine cervix tumor, } 10 \text { spots of CIN, } 10 \text { spots of inflammation and } \\
10 \text { adjacent normal tissue }\end{array}$ \\
\hline TE803 & Testis & $\begin{array}{l}\text { Testis disease spectrum (testicular cancer progression) tissue microarray, } \\
\text { containing } 26 \text { spots of seminoma, } 12 \text { embryonal carcinoma, } 10 \text { yolk sac tumor, } \\
7 \text { teratoma, } 4 \text { tuberculosis, } 6 \text { atrophy, } 10 \text { adjacent normal tissue and } 5 \text { normal tissue }\end{array}$ \\
\hline
\end{tabular}

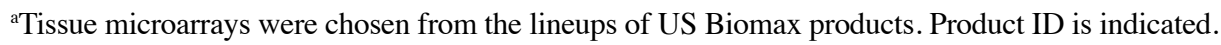

FAM107A has been regarded as a candidate of tumor suppressor gene (TSG) because of its decreased expression in cancer and because of the fact that introduction of FAM107A suppresses cancer cell proliferation and induces apoptosis (7,10-13). In contrast, it was recently reported that FAM107A is highly expressed in the invasive component of gliomas and that it drives tumor invasion by functioning as a new cytoskeletal crosslinker that regulates focal adhesion dynamics and cell movement $(14,15)$. Consequently, the physiological role and function of FAM107A as a TSG remain controversial.

In humans, FAM107B protein is encoded by the gene on chromosome $10 \mathrm{p} 13$, sharing $65 \%$ sequence similarity with FAM107A in their N-terminal DUF1151 regions. We previously investigated histopathological localization and functions of FAM107B in gastrointestinal cancers, and designated it as heat-shock-inducible tumor small protein (HITS) (16) based on its unique expression pattern and biological properties in cancer cells and its low molecular weight (18 kDa) among a family of heat-shock proteins (HSPs) (17-19). Similar to FAM107A, HITS expression is decreased or absent in gastric and colorectal cancers; moreover, it inhibits tumor cell proliferation in vitro (16), which contrasts with previously described oncogenic activities of other HSPs such as HSP70 and HSP90 (20-24).

In this study, we performed immunohistochemical examination of HITS expression in cancer tissues of the multiple organs spotted on tissue microarrays that include clinical and histopathological information of the respective tumor samples. Consistent with the hypothesis that HITS is a tumor suppressor protein in gastrointestinal cancer (16), our current study demonstrated that loss of HITS expression is commonly observed in cancers of multiple organs and involved in tumor proliferation.

\section{Materials and methods}

Tissue microarrays. Several types of human tissue microarrays including normal, malignant, and metastatic formats (US Biomax, Inc., Rockville, MD) were used to examine HITS expression in cancer and to compare the results with clinical and histopathologic characteristics of the tumors (Table I). The high-density multiple organ tumor and normal tissue microarray (MC5003) was used for the initial screening of HITS expression. This microarray includes 18 types of tumor $(20$ cases/each tumor type) and normal tissues (5 cases/each organ) with TNM classification and pathology grades of the tumors. According to results of HITS expression in MC5003 tissue microarray and statistical analysis, additional analysis of individual organs was used with the following tissues microarrays. BR1503 is a breast cancer tissue microarray that includes TNM classification and pathology grades of the respective tumor spots and the levels of immunohistochemical expression of human epidermal growth factor receptor 2 (HER2), estrogen receptor (ER), progesterone receptor (PR), p53 and Ki-67. BRM961 is a breast cancer tissue microarray including the spots of primary and matched metastatic tumors in lymph nodes and adjacent normal breast tissues. TH807 includes tissue spots of various thyroid diseases. CR602 includes the tissue spots of uterine cervical diseases [cervical cancer, cervical intraepithelial neoplasia (CIN), inflammation] and adjacent normal tissues, and TE803 includes those of testis diseases (testicular benign and malignant tumors).

Antibodies. To generate a polyclonal anti-human HITS antibody, rabbits were immunized with keyhole limpet hemocyanin (KLH)-conjugated polypeptides (MAEPDYIEDDNPE) as an antigen (16). The antibody was purified from the anti-serum using affinity chromatography against the same polypeptides 


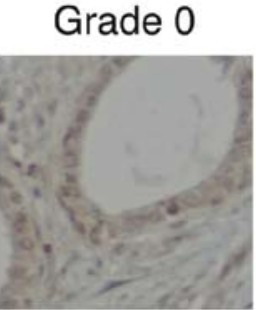

Prostate

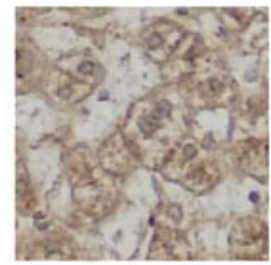

Liver

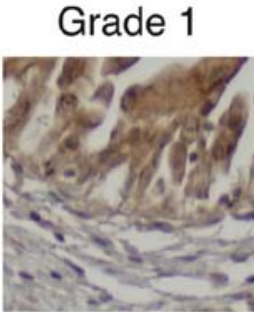

Bladder

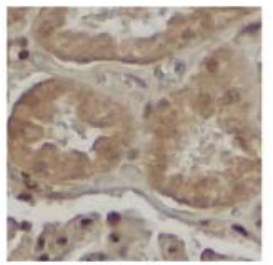

Kindey

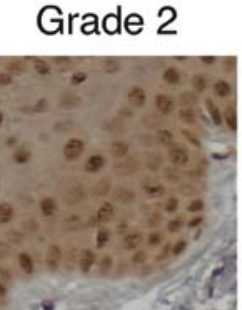

Skin

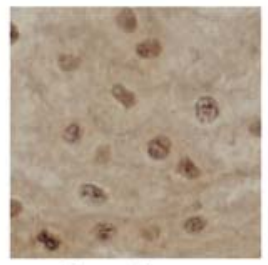

Esophagus

\section{Grade 3}

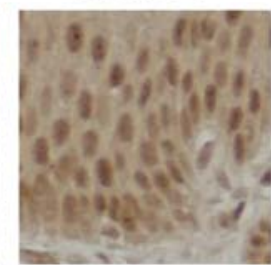

Uterine Cervix

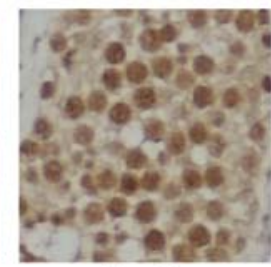

Testis
Grade 4

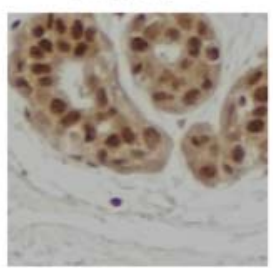

Breast

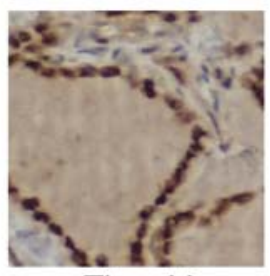

Thyroid

Figure 1. Scoring of HITS expression. Immunohistochemical evaluation of HITS expression was classified into grades 0-4 according to its signal intensity and nuclear localization. Representative findings of the indicated scores of HITS expression in the respective normal tissues are shown.

cross-linked to agarose beads, and verified its specificity by flow cytometry of immunostaining and western blot analysis. Antibodies to $\beta$-actin (Santa Cruz Biotechnology, Santa Cruz, $\mathrm{CA}$ ), proliferating cell nuclear antigen (PCNA) and survivin (Dako A/S, Glostrup, Denmark) were purchased.

Immunohistochemistry. Expression and localization of HITS, PCNA and survivin in paraffin sections of tissues and microarrays were examined using immunohistochemical staining, as conducted in a previous study (25). Representative paraffin sections and tissue microarrays placed on silanized slides were treated by microwaving in the citrate buffer to unmask antigens. Then they were incubated with $0.3 \% \mathrm{H}_{2} \mathrm{O}_{2}$ in methanol and with $10 \%$ normal goat serum for blocking endogenous peroxidase activity and nonspecific antibody binding. The pretreated sections were incubated with the respective antibodies (1:500 dilution each) overnight at $4^{\circ} \mathrm{C}$ with subsequent serial incubation with the biotinylated goat anti-rabbit IgG (Vector Laboratories Inc., Burlingame, CA) and standard avidin-biotinperoxidase complex (ABC). Cell nuclei were counterstained with hematoxylin.

Scores for HITS expression and statistical analysis. We assigned grades 0-4 to microscopic evaluation of HITS expression in tissues according to the signal intensity and nuclear localization of HITS (Fig. 1). Grade 0, no staining in cytoplasm and nucleus; grade 1, borderline cytoplasmic staining but no apparent nuclear localization; grade 2, weak nuclear staining; grade 3, moderate nuclear staining; grade 4, strong nuclear staining.

Scores of HITS grading in the respective groups of tissue specimens were expressed as means \pm standard deviation (SD). The mean scores of HITS expression of the two groups were compared using two-sided Mann-Whitney U tests. For comparison of the scores of more than two groups, the significance of differences among the data was determined using one-way ANOVA followed by Scheffe's F post hoc test. Correlation between scores of HITS expression and the clinical and pathological parameters of the tumors was determined using the nonparametric Spearman's rank correlation analysis. Values of $\mathrm{P}<0.05$ were considered significant.

In breast cancer, desmoplastic reaction, also called reactive fibrosis, is characterized by mobilization of fibroblasts and deposition of abundant collagen as a stromal response to an invasive carcinoma. The HITS expression scores for breast cancers with and without desmoplastic stromal reaction were compared statistically.

In vivo tumorigenicity assays. We previously established a human uterine cervical-cancer-derived HeLa Tet-On advanced cell line (Clontech Laboratories, Inc., Mountain View, CA) transduced with a mock or Tet-inducible HITS gene (16). Cells $\left(1 \times 10^{6}\right)$ of each HeLa cell line were inoculated subcutaneously to two opposite sites of flank in each Scid mouse ( $n=14$; Charles River Laboratories Japan Inc., Yokohama, Japan). These mice were divided into two groups and treated or not treated with doxycycline $(1.5 \mathrm{mg} / \mathrm{ml}$; Clontech Laboratories Inc.) dissolved in drinking water in light-protected bottles, respectively, for 8 weeks. Following treatment, all mice were euthanized. Tumors removed from mice were weighed and fixed in neutral-buffered $10 \%$ formalin and embedded in paraffin for histopathologic and immunohistochemical examination. Tumor formation in mice with $(n=7)$ or without $(n=7)$ doxycycline administration was expressed as average weight \pm SD. The statistically significant difference in tumor formation between the groups was determined using a two-sided Mann-Whitney U test, for which $\mathrm{P}<0.05$ was considered significant. We repeated this experiment twice to confirm reproducibility. The animal study was conducted according to the Guidelines for Experimental Animals at Kanazawa Medical University and the National Guidelines for Animal Usage (http://www.lifescience.mext. go.jp/policies) in Japan.

\section{Results}

Screening for the expression of HITS in multiple tissues. The high-density multiple organ tumor and normal tissue array 
A

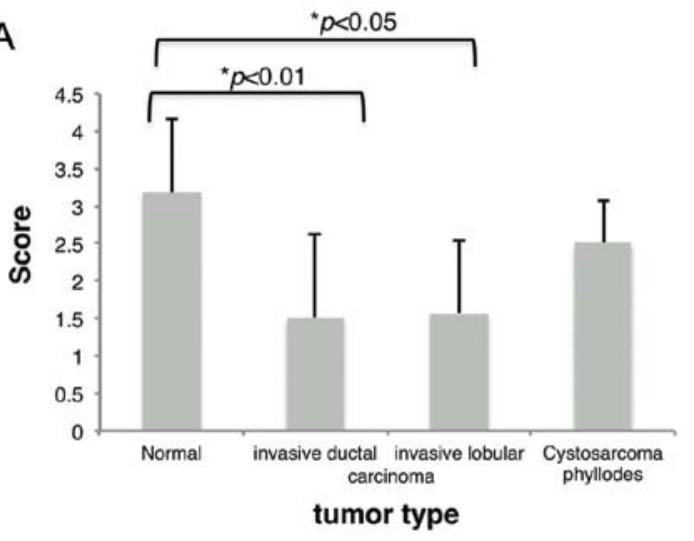

B

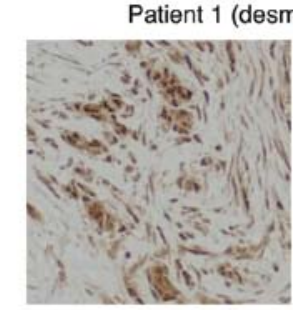

Primary tumor

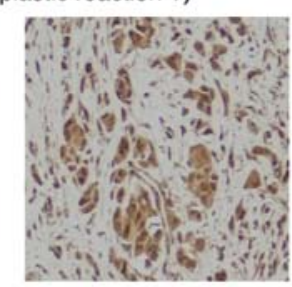

LN metastasis
Patient 2 (desmoplastic reaction -)

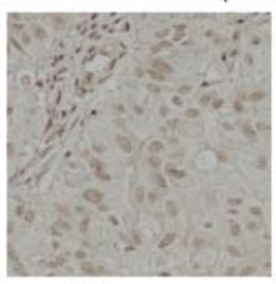

Primary tumor

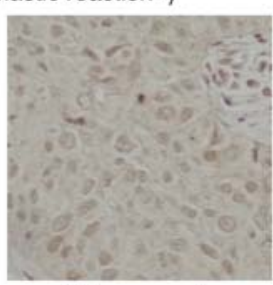

LN metastasis
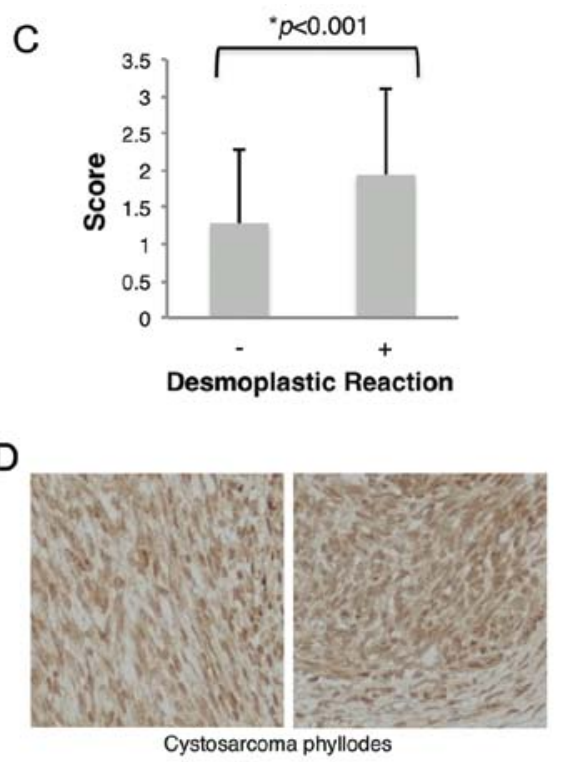

$\mathrm{E}$

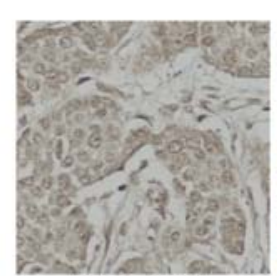

PR +++, HER2 -

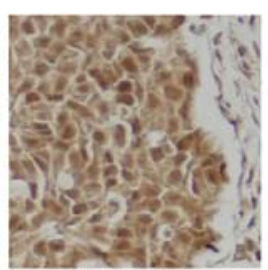

PR - HER2 +++ $100 \mu \mathrm{m}$

Figure 2. HITS expression in breast tumors. (A) Scores of HITS expression in tissue microarrays MC5003, BR1503 and BRM961 are summed up in normal breast tissues $(n=23)$, invasive ductal carcinoma $(n=177)$, invasive lobular carcinoma $(n=7)$ and cystosarcoma phyllodes $(n=4)$, respectively, and are expressed as means \pm SD and compared by Scheffe's F post hoc test. (B) HITS expression was compared between the primary tumor and lymph node metastasis in the patient 1 or 2 with or without desmoplastic reaction, respectively. (C) Breast cancers were classified into two groups with (n=67) and without (n=119) desmoplastic reaction. The scores of HITS expression of the two groups were compared. Statistical analysis was performed using two-sided Mann-Whitney U test. Representative staining with (D) IHC of HITS in cystosarcoma phyllodes and (E) PR(+++), HER2(-) or PR(-), HER2(+++) invasive ductal carcinoma is shown. * Denotes a statistically significant effect $(\mathrm{p}<0.05$, bracket).

(MC5003) including tumors of 18 types along with corresponding normal tissues was used for immunohistochemical screening for the expression of HITS. Scores of HITS expression in normal $(\mathrm{n}=5)$ and cancer tissues $(\mathrm{n}=20)$ in each organ are added up separately, expressed as mean $\pm \mathrm{SD}$, and compared statistically using the Mann-Whitney U test. Despite the small number of samples in each group, lower expression of HITS in tumor tissues than their normal counterparts was frequent to a significant degree in cancers of the thyroid, breast, colon, and uterine cervix (Table II). Scores of HITS expression in cancer tissues seemed lower than their normal counterparts in the lung, stomach, and testis, although no statistically significant differences were found among them. In contrast, in the tumors of the liver, pancreas, ovary, and urinary tracts including the kidney, bladder, and prostate, scores of HITS expression in cancer tissues seemed slightly higher than those in the corresponding normal tissues, although the intensity of HITS expression in these organs was very low, even in normal tissues (Table II and Fig. 1).
Through screening of the MC5003 tissue array, we selected four organs of the breast, thyroid, testis and uterine cervix for the next step of investigation of detailed pathological analysis and statistical analysis using cancer tissue arrays of the respective organs to explore HITS expression in the tumors (Table I).

HITS expression in breast cancer. Because HITS expression was high in normal breast tissues (Table II and Fig. 1), average scores of HITS expression summed up in the normal tissues spotted on the three tissue-microarrays MC5003, BR1503 and BRM961 were $3.17 \pm 0.98$. The mean score of HITS expression in cancer tissues was $1.50 \pm 1.08$, which was significantly lower than in normal tissues $(\mathrm{p}<0.001)$. Decreased HITS expression was common to the two representative histological types of breast cancers, invasive ductal and lobular carcinomas (Fig. 2A). Comparison of cancers with different stromal reactions revealed that the breast cancers associated with desmoplastic reaction exhibited significantly higher HITS expression than those without this stromal reaction (Fig. 2B and C). Cystosarcoma 
Table II. Immunohistochemical screening for the expression of HITS in multiple organs and tissues.

\begin{tabular}{lccr}
\hline & Normal $^{\mathrm{a}}(\mathrm{n}=5)$ & Cancer $^{\mathrm{a}}(\mathrm{n}=20)$ & P-value $^{\mathrm{b}}$ \\
\hline Brain & $2.20 \pm 1.30$ & $2.70 \pm 1.34$ & 0.43 \\
Head and neck & $2.80 \pm 1.10$ & $2.67 \pm 0.84$ & 0.61 \\
Thyroid & $3.80 \pm 0.45$ & $1.30 \pm 0.73$ & $<0.01$ \\
Lung & $3.00 \pm 1.00$ & $2.25 \pm 1.12$ & 0.18 \\
Breast & $4.00 \pm 0.00$ & $2.40 \pm 1.19$ & $<0.01$ \\
Esophagus & $2.20 \pm 1.30$ & $1.55 \pm 1.10$ & 0.28 \\
Stomach & $2.33 \pm 1.15$ & $1.31 \pm 1.08$ & 0.13 \\
Colon & $3.20 \pm 0.45$ & $1.42 \pm 0.69$ & $<0.01$ \\
Liver & $0.60 \pm 0.55$ & $0.75 \pm 0.44$ & 0.51 \\
Pancreas & $1.20 \pm 0.45$ & $1.58 \pm 0.96$ & 0.51 \\
Uterus & $1.60 \pm 1.34$ & $1.84 \pm 1.12$ & 0.42 \\
Uterine cervix & $3.80 \pm 0.45$ & $1.68 \pm 0.89$ & $<0.01$ \\
Ovary & $1.60 \pm 1.14$ & $1.80 \pm 1.01$ & 0.80 \\
Kidney & $0.80 \pm 0.45$ & $1.35 \pm 1.42$ & 0.64 \\
Bladder & $2.00 \pm 1.41$ & $2.75 \pm 1.12$ & 0.24 \\
Prostate & $0.80 \pm 0.84$ & $1.63 \pm 1.21$ & 0.15 \\
Testis & $3.60 \pm 0.55$ & $2.21 \pm 1.23$ & 0.06 \\
Soft tissue & $3.40 \pm 0.55$ & $3.35 \pm 0.88$ & 0.82 \\
Skin & $3.00 \pm 0.00$ & $2.40 \pm 1.18$ & 0.29 \\
Lymph node & $2.20 \pm 0.84$ & $1.70 \pm 0.92$ & 0.23 \\
\hline
\end{tabular}

${ }^{a}$ HITS expression scores of normal $(n=5)$ and cancer tissues $(n=20)$ in each organ are added separately (tissue array: MC5003). Data are the mean $\pm \mathrm{SD}$. ${ }^{\mathrm{b}} \mathrm{S}$ cores are compared using the Mann-Whitney $\mathrm{U}$ test; $\mathrm{P}<0.05$ was considered statistically significant.

phyllodes is a rare, usually large, fast growing breast tumor that arises from periductal cells of the breast; it is regarded as a borderline malignancy (26). This tumor showed an intermediate score of HITS expression between normal and carcinoma tissues (Fig. 2A and D).

The relation between tumor progression and HITS expression was examined according to the TNM classification. In the results of statistical analysis presented in Table III, the HITS expression scores were lower in accordance with advances in the TNM stages of tumors ( $r s=-0.19, \mathrm{p}=0.01)$. They were inversely correlated with the T-value (primary tumors) but not with the $\mathrm{N}$-value (lymph node metastasis). To support this, the intensity of HITS expression showed no difference between the primary and metastatic lymph node tumors in the same patients $(1.37 \pm 1.17$ and $1.23 \pm 0.94$, respectively, $p=0.40)$ (Fig. $2 B)$. No relation to the histological grade of tumor differentiation was found (Table III).

Although the most important prognostic factors are the tumor size, histological grade and lymph node stage (27), the importance of several molecular markers in breast cancer has been of considerable interest recently, not only as prognostic markers, but also as predictors of response to therapy (28). For further analysis of correlation between HITS expression and other pathological parameters, we exploited the tissue microarray BR1503, which includes data of immunohistochemical expression of HER2, ER, PR, p53 and Ki-67 in addition to those of TNM stages and
Table III. Expression of HITS for breast cancer.

\begin{tabular}{|c|c|c|c|}
\hline Parameter & HITS mean \pm SD $(n)$ & rs & P-value \\
\hline TNM stage ${ }^{a}$ & & -0.19 & 0.01 \\
\hline I & $1.71 \pm 0.91$ & & \\
\hline IIa & $1.68 \pm 1.15 \quad(92)$ & & \\
\hline $\mathrm{IIb}$ & $1.27 \pm 1.07$ & & \\
\hline IIIa & $1.47 \pm 1.22$ & & \\
\hline IIIb & $1.07 \pm 0.80$ & & \\
\hline $\mathrm{T}^{\mathrm{a}}$ & & -0.23 & $<0.01$ \\
\hline 1 & $1.71 \pm 0.91$ & & \\
\hline 2 & $1.67 \pm 1.17(126)$ & & \\
\hline 3 & $1.03 \pm 0.87$ & & \\
\hline 4 & $1.07 \pm 0.55$ & & \\
\hline $\mathrm{N}^{\mathrm{a}}$ & & -0.05 & 0.48 \\
\hline 0 & $1.55 \pm 1.10(132)$ & & \\
\hline 1 & $1.34 \pm 1.09$ & & \\
\hline 2,3 & $1.63 \pm 1.20$ & & \\
\hline $\mathrm{ER}^{\mathrm{b}}$ & & 0.02 & 0.82 \\
\hline$-\sim+$ & $1.43 \pm 1.04 \quad(88)$ & & \\
\hline$++\sim+++$ & $1.37 \pm 1.02$ & & \\
\hline $\mathrm{PR}^{\mathrm{b}}$ & & -0.22 & $<0.05$ \\
\hline$-\sim+$ & $1.55 \pm 1.02 \quad(87)$ & & \\
\hline$++\sim+++$ & $1.14 \pm 1.00$ & & \\
\hline $\mathrm{TP} 3^{\mathrm{b}}$ & & -0.06 & 0.47 \\
\hline$-\sim+$ & $1.45 \pm 1.08 \quad(85)$ & & \\
\hline$++\sim+++$ & $1.34 \pm 0.93$ & & \\
\hline $\mathrm{HER}{ }^{\mathrm{b}}$ & & 0.23 & $<0.01$ \\
\hline$-\sim+$ & $1.27 \pm 1.20$ & & \\
\hline$++\sim+++$ & $1.48 \pm 0.94 \quad(90)$ & & \\
\hline $\mathrm{Ki}-67^{\mathrm{b}}$ & & 0.33 & $<0.001$ \\
\hline$-\sim+$ & $1.27 \pm 1.28$ & & \\
\hline$++\sim+++$ & $1.46 \pm 0.94(102)$ & & \\
\hline Histological grade ${ }^{c}$ & & 0.006 & 0.93 \\
\hline 1 & $2.17 \pm 1.17$ & & \\
\hline 2 & $1.38 \pm 1.07$ (134) & & \\
\hline 3 & $1.68 \pm 1.09$ & & \\
\hline $\mathrm{Age}^{\mathrm{c}}$ & & 0.13 & 0.07 \\
\hline$<49$ & $1.36 \pm 1.08(105)$ & & \\
\hline$>50$ & $1.64 \pm 1.08 \quad(90)$ & & \\
\hline
\end{tabular}

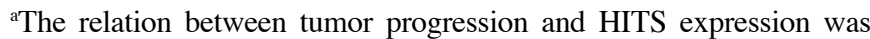
examined according to the TNM classification and investigated using nonparametric Spearman rank correlation analysis (tissue arrays: MC5003, BR1503, BRM961). ${ }^{\mathrm{b}}$ Correlation between HITS expression and pathological parameters was investigated: HITS expression scores were collected in each grade of IHC results $(-,+,++,+++)$ of HER2, ER, PR, P53 and Ki-67 described in tissue array BR1503. Data were statistically compared using the nonparametric Spearman's rank correlation analysis. Scores of HITS expression were summed up separately in $-\sim+$ or $++\sim+++$ of HER2, ER, PR, P53 and Ki-67 expression, and are shown as means $\pm \mathrm{SD}$ (tissue array: BR1503). ${ }^{\text {CT }}$ The relation to histological grade and age was investigated. Histological grades 1-3 in pathology diagnosis are, respectively, equivalent to well differentiated, moderately differentiated, and poorly differentiated (The American Joint Commission on Cancer; http://www.cancerstaging.org/). HITS expression scores in each grade or generation and statistical results with the nonparametric Spearman's rank correlation analysis are shown (tissue arrays: MC5003, BR1503, BRM961). 


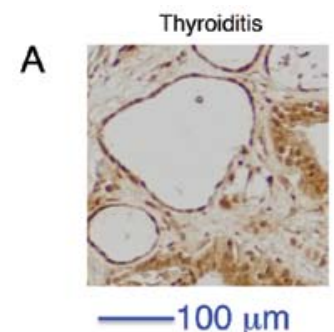

B

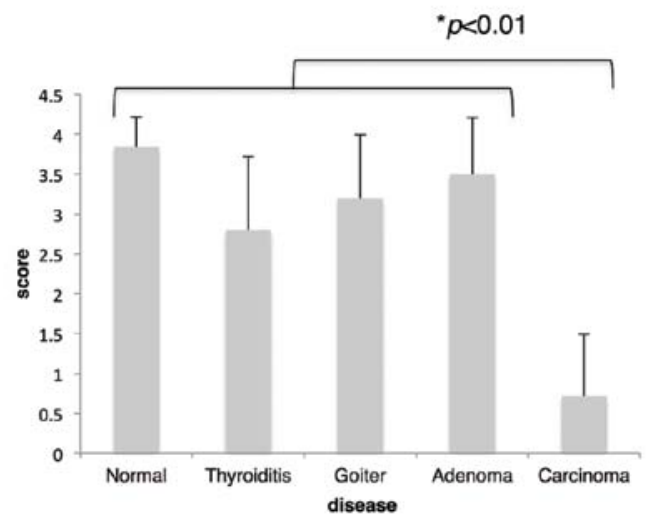

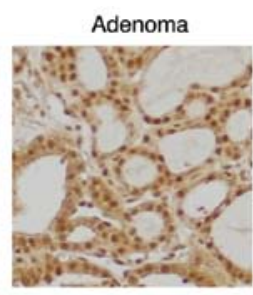
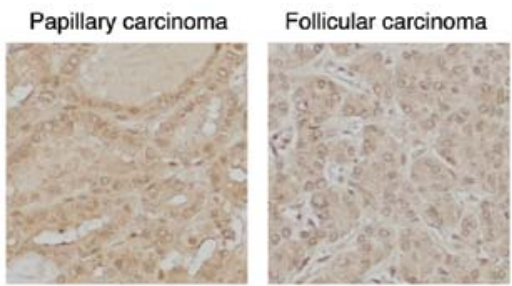

C

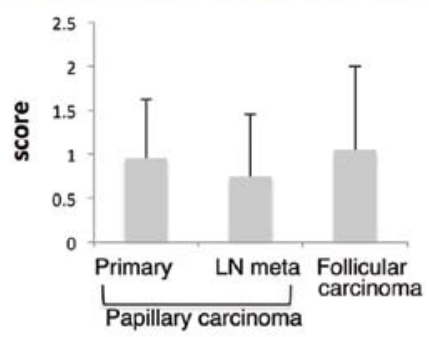

D

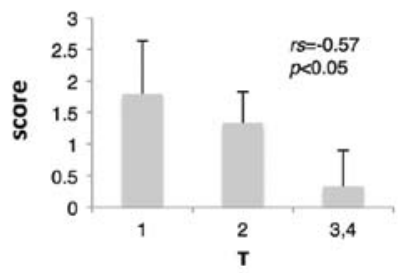

Figure 3. HITS expression in thyroid diseases. (A) Representative findings of HITS expression in the different thyroid diseases. (B) Scores of HITS expression in the tissue microarrays MC5003 and TH807 are summed up in normal thyroid tissues $(n=24)$, thyroiditis $(n=10)$, goiter $(n=10)$, adenoma $(n=10)$ and carcinoma $(\mathrm{n}=41)$, respectively, and are shown as the mean \pm SD. Statistical analysis using Scheffe's F post hoc test indicates a significant difference in HITS expression scores between carcinoma and normal or benign thyroid diseases. (C) Comparison of HITS expression between the primary tumors and lymph node metastasis of papillary carcinoma ( $\mathrm{n}=11)$ and follicular carcinoma $(\mathrm{n}=10)$ in the tissue array TH807 are shown. (D) Inverse correlation between HITS expression scores and the T-value of the TNM grading were revealed by nonparametric Spearman's rank correlation analysis ( $(r s=-0.57, p<.05)$. The numbers of samples were $T 1(n=5), T 2(n=12)$ and T3 or $4(\mathrm{n}=3)$. "Denotes a statistically significant effect ( $\mathrm{p}<.05$, bracket).

A
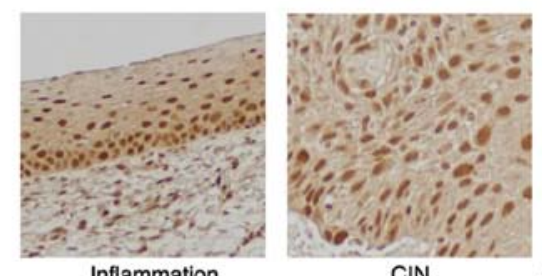

$\mathrm{CIN}$

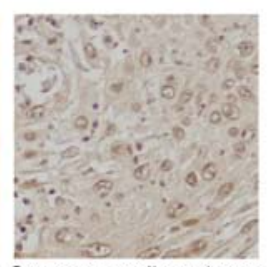

Squamous cell carcinoma
C
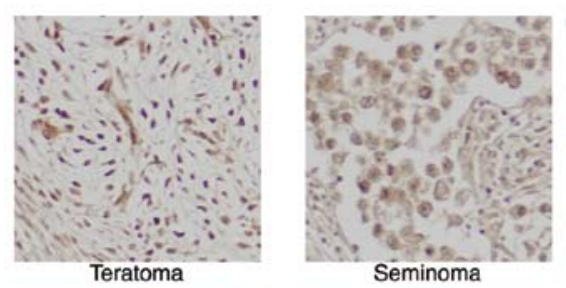

$-100 \mu \mathrm{m}$
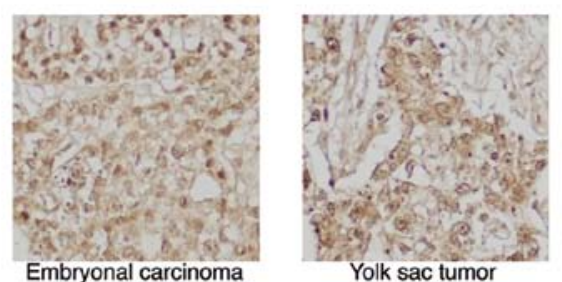

B

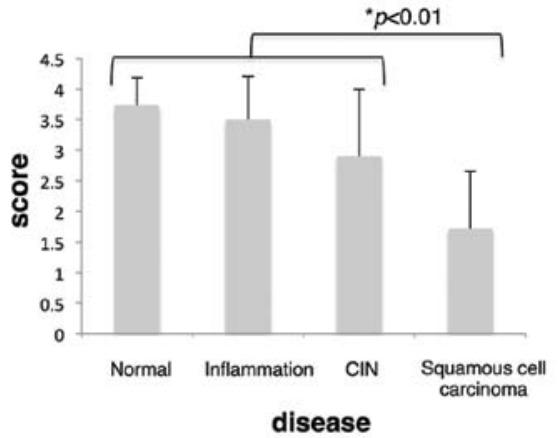

D

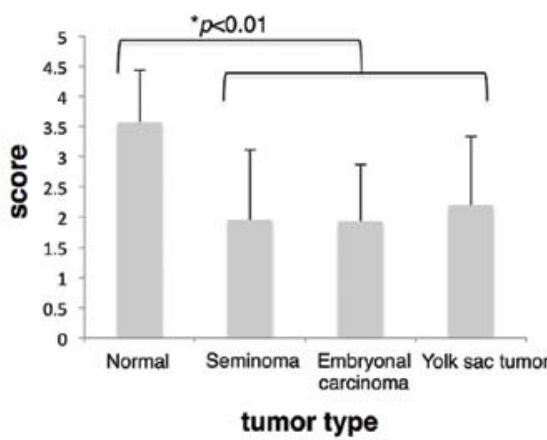

Figure 4. HITS expression in the uterine cervical tissues and the testicular tumors. (A) Representative staining patterns of HITS in various cervical diseases. (B) HITS expression scores in normal tissues $(n=15)$, lesions with inflammation $(n=10)$, CIN $(n=10)$ and invasive squamous cell carcinoma $(n=49)$ of the uterine cervix on the tissue microarrays MC5003 and CR602 are shown as the mean \pm SD. They are statistically compared using Scheffe's F post hoc test. (C) Representative findings of HITS expression in benign teratoma and malignant tumors of the testis. (D) Scores of HITS expression in normal testis $(n=26)$, seminoma $(n=41)$, embryonal carcinoma $(n=16)$ and yolk sac tumor $(n=10)$ on the tissue microarrays MC5003 and TE803 were compared statistically using Scheffe's $F$ post hoc test. "Denotes a statistically significant effect $(\mathrm{p}<0.05$, bracket). 
pathology grade (Table I). Statistical analysis revealed that the score of HITS expression was correlated positively with the expressions of HER2 ( $\mathrm{rs}=0.23, \mathrm{p}<0.01$ ) and $\mathrm{Ki}-67$ ( $\mathrm{rs}=0.33$, $\mathrm{p}<0.001$ ), but inversely with PR expression ( $\mathrm{rs}=-0.22, \mathrm{p}<0.05)$ (Table III). For example, the average score of HITS expression in cancers showing PR- and HER2+++ was 2.16 $\pm 0.99(n=25)$, although the score of HITS expression in the two cases with $\mathrm{PR}+++$ and HER2- was grade $0(\mathrm{p}=0.02)$ (Fig. 2E). Among other pathological parameters, age has been shown to be a prognostic factor: young patients ( $<35$ years) with breast cancer have a poorer prognosis (29). The present study showed an apparent trend in the increase of HITS scores in cancer tissue in accordance with age, although it was not statistically significant $(r s=0.13, p=0.07)$ (Table III). Means of HITS expression scores in normal breast tissues were greater than 3.0 in all generations (data not shown).

HITS expression in the thyroid, uterine cervical and testicular diseases. In analysis of the thyroid disease tissue microarray (TH807), the HITS expression intensity clearly distinguished thyroid cancer (papillary and follicular carcinomas) from normal tissue, thyroiditis, diffuse goiter, and adenoma (Fig. 3A and B). Consistent with the results obtained for breast cancer, the HITS expression level was similar in primary tumor and lymph node metastasis in the same patients, but inversely correlated with the T-values of TNM grading (Fig. 3C and D).

Regarding analysis of the uterine cervical disease tissue microarray (CR602), HITS expression was found in cervical epithelial cell nuclei in inflammation and CIN, but it was markedly decreased in invasive squamous cell carcinoma (Fig. 4A and $\mathrm{B}$ ). Infection of human papilloma virus (HPV) is known as an inducer of cervical cancers because of strong causal relations between HPV infection, CIN and invasive carcinoma (30). Because some lesions among those of CIN progress to invasive cancer in 10 to 20 years (31), our results suggest that HITS expression is lost during the progression of CIN toward invasive carcinoma.

Analysis of the testis disease tissue microarray (TE803) showed that, similar to normal breast, thyroid and uterine cervix tissues, HITS expression was high in normal testis (average score >3.5) (Table II, Figs. 1 and 4D). Tumors of the testis, including germ cell tumors (seminoma) and embryonic tumors (embryonal carcinoma and yolk sac tumor), showed significantly lower expression of HITS than either normal testis or benign teratoma (Fig. 4C and D). No significant difference was found in expression levels of HITS between seminoma and non-seminomatous tumors (embryonal carcinoma and yolk sac tumor).

Inducible expression of HITS in vivo reduces tumor size. Based on the immunohistochemical analysis of breast and thyroid cancer tissue microarrays showing that intensity of HITS expression is inversely correlated with the T-value of TNM grading, i.e. primary tumor size, we hypothesized that tumor proliferation might be inhibited by ectopic or forced expression of HITS, which functions as a tumor suppressor protein. Previously, we reported that tetracycline-inducible expression (Tet-ON) of HITS in cancer cells diminished proliferation in response to growth factors such as epidermal growth factor, fibroblast growth factor and fetal calf serum (16). To investigate the role of HITS in tumor proliferation in vivo, HITS-transduced and mock-transduced human cervical cancer HeLa cells were transplanted subcutaneously in the flank of Scid mice. Then their tumor formation status was observed for 8 weeks with or without induction of HITS by doxycycline administration in drinking water. As portrayed in Fig. 5A, all mock-transduced cells developed tumors, whereas the Tet-inducible HITS-transduced cells generated tumors in the absence of HITS induction, indicating the suppressive role of HITS for tumor growth. The HITS-induced tumors, although very rarely generated (only one out of seven) and small (Fig. 5A), were processed for histological examination. Regarding pathological examination of the tumors, HITS-induced tumors showed no morphologically distinct gross or microscopic differences except for tumor size (Fig. 5A and B). Immunohistochemical staining of PCNA and survivin showed no apparent difference in intensities between tumors with and without HITS induction, although the area of positively stained cells surrounding central necrosis was much less in HITS-induced tumors than in non-induced tumors, which might reflect the minimal size of tumor formation (Fig. 5C).

\section{Discussion}

In this study, we clarified that loss of HITS expression in cancer is common among organs in which the expression level of HITS was high in respective normal tissues. Statistical analysis of data obtained from tissue microarray examination revealed the relevance of HITS expression scores to several pathological parameters, especially to primary tumor size (Table III and Fig. 3D). Forced induction of HITS in cancer cells inhibited the proliferation of tumor xenografts in rodents. These results suggest that HITS might be a tumor suppressor in breast, thyroid, uterine cervix and testis as well as stomach and colon (16).

Both FAM107A (TU3A/DRR1) and FAM107B (HITS) are expected to be tumor suppressors because their expressions are often decreased in cancer cells; moreover, their introduction suppresses cancer cell growth $(6,7,10,16,32)$. HITS possesses a conserved domain of DUF1151 in the N-terminal region, as does FAM107A. However the tissue distribution and the physiological function of HITS are distinctive. First, HITS is expressed in various tissues including digestive, respiratory, genital, and lymphoid organs (Table II and Fig. 1) (16). In contrast, FAM107A is expressed predominantly in the nervous system, especially in neurons, but not in astrocytes or in oligodendrocytes $(13,14)$. Regarding subcellular localization, both HITS and FAM107A are localized in the cell nucleus because of a nuclear localization signal in the center of the protein sequences (16). Second, the HITS gene carries the promoter region providing heat-shock transcription factor 1 (HSF1) binding sites and the transcription of HITS is amplified by heat-shock or hyperthermia treatment (16). This is a salient distinguishing feature of HITS because other HSPs and HSF1 are regarded as having oncogenic activities in many respects (22-24,33-37). In our studies, similarly to FAM107A, ectopic or forced expression of HITS in cancer cells inhibited tumor growth in vitro and in vivo (Fig. 5) (16). Consequently, HITS is a potential tumor suppressor protein with a unique feature of its transcriptional induction by heatshock stimulation. It is expected to be useful for tumor diagnosis and for monitoring therapeutic effects of hyperthermia.

A previous study by the authors found that the level of HITS expression in gastrointestinal cancer cells was lower 
A Doxycycline OFF

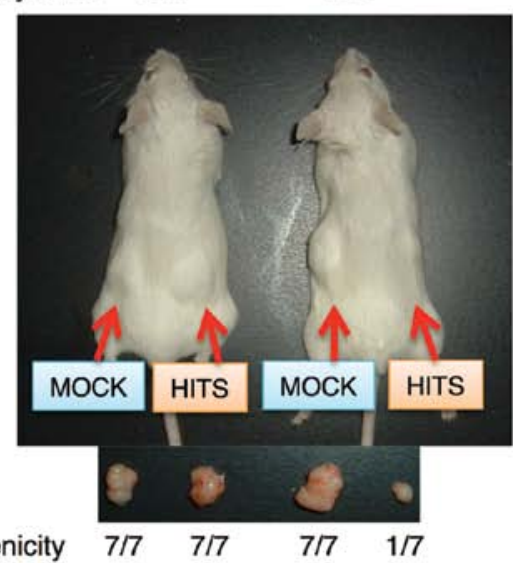

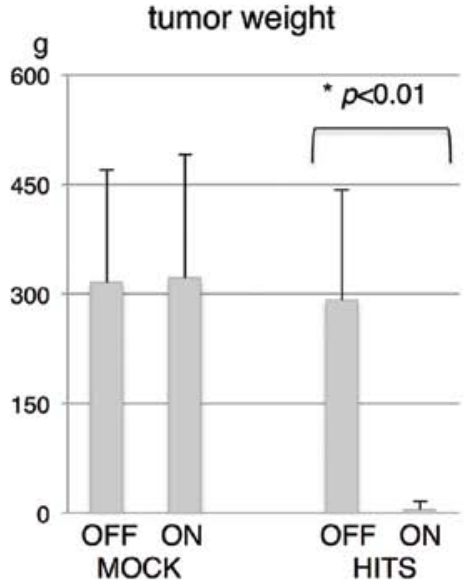

B
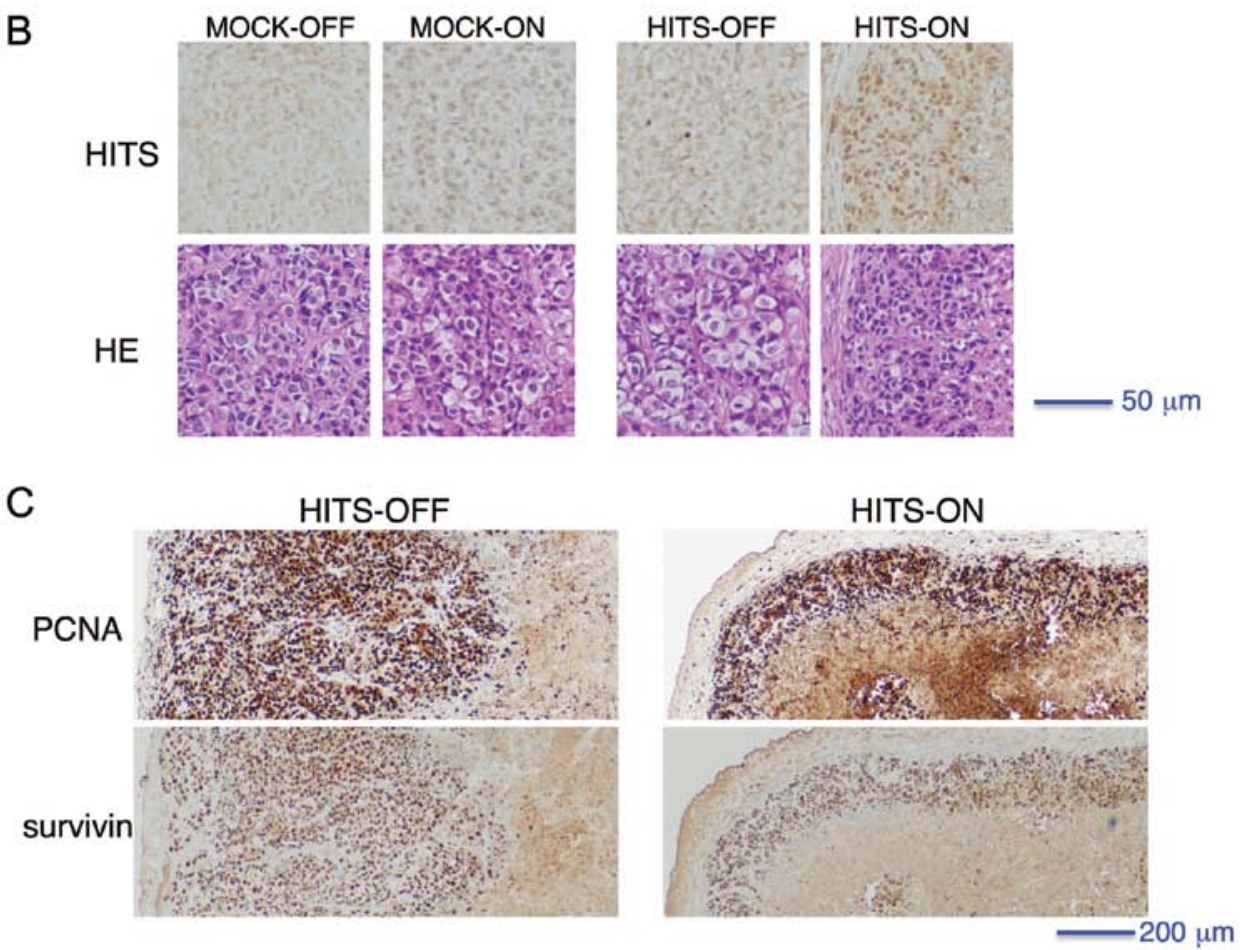

Figure 5. Effects of forced expression of HITS in the uterine cervical cancer xenograft model. (A) In vivo tumorigenicity was assayed by inoculating $1 \times 10^{6}$ HeLa cells transduced with mock or Tet-inducible HITS in Scid mice, with (mouse on the right) or without (mouse on the left) doxycycline administration. Their tumorigenicity was examined at 8 weeks after the respective treatments and is expressed as averages of xenograft weight \pm SD (graph). Significance of the difference was determined using two-sided Mann-Whitney U tests. (B and C) Tumors removed from mice were fixed and processed for histopathological and immunohistochemical examinations. (B) HE staining (lower panels) and immunohistochemical staining of HITS (upper panels). (C) Immunohistochemical staining of survivin (lower panels) and PCNA (upper panels). "Denotes a statistically significant effect ( $\mathrm{p}<0.05$, bracket).

than in normal epithelial cells, although its expression pattern and intensity varied among cancers of different histological types (16). Expression of HITS was decreased during the process of colorectal adenoma-to-carcinoma sequence, and also decreased in intestinal-type gastric adenocarcinomas, but not in diffuse type adenocarcinomas. HITS expression was positive in both stomach and colon mucinous adenocarcinomas. In the present study, tissue microarray analysis revealed that loss of HITS expression in cancer was commonly observed in several organs in a histopathological-type-specific manner. HITS expression was decreased in the prevalent histologic types of breast cancer, invasive ductal, and lobular carcinomas compared with normal breast ducts (Fig. 2A). Statistical analysis elucidated that HITS expression became lower in accordance with breast cancer progression on TNM staging (Table III). It is particularly interesting that, among the factors determining TNM stages, HITS expression scores were inversely correlated with the $\mathrm{T}$-value but not with the $\mathrm{N}$-value. Inverse association with the T-value was also found in thyroid cancer (Fig. 3D).

HITS expression was significantly higher in cancers with a desmoplastic stromal reaction (Fig. 2B and C), similar to diffuse-type gastric cancers with scirrhous stromal reaction that exhibited much higher HITS expression than intestinaltype cancers (16). In further statistical analysis with other pathological parameters of breast cancer, the score of HITS expression correlates positively with expression of HER2 and 
Ki-67, and inversely with PR expression (Table III and Fig. 2E). These molecular markers play important roles in carcinogenesis and tumor progression in breast cancers (38-40). Expression levels of HER2, ER and PR are well-established markers for predicting anti-tumor effects of trastuzumab (Herceptin), a therapeutic monoclonal antibody to HER2 and of endocrine therapy, respectively $(41,42)$. Scores of HITS expression are apparently high in HER2 positive, Ki-67 positive, PR negative, and desmoplastic reaction-positive breast cancer, which was thought to be an aggressive phenotype presenting increased risk of disease recurrence and shortened survival $(43,44)$.

Invasive ductal carcinoma of the breast is classified histologically into two subtypes: pure invasive ductal carcinoma (IDC) and IDC associated with ductal carcinoma in situ (IDC/DCIS) (40). Pure IDC is morphologically, pathologically, and genetically distinct and has a worse prognosis than IDC/DCIS, suggesting that they might be different biological and clinical tumor entities $(33,45-47)$. HER2 amplification and $\mathrm{Ki}-67$ expression were significantly higher in pure IDC cases than in IDC/DCIS, although the PR expression in pure IDC was significantly lower than in IDC/DCIS. The ER expression and p53 expression showed no statistically significant differences between the two histological subtypes (40). The correlation of HITS expression with the molecular characteristics as described above suggests that the distinct staining pattern of HITS can surrogate the reported difference in molecular characteristics between pure IDC and IDC/DCIS, thereby enabling differential diagnosis of these tumor subtypes with different clinical outcomes.

Considering our results that HITS expression was lost during the course of tumor progression in T-value of TNM grading (Table III and Fig. 3D), it is speculated on one hand that HITS expression decreases during the long process from pre-neoplastic or earlier neoplastic lesions such as DCIS in breast, intestinal metaplasia in stomach, tubular adenoma in colon and CIN in uterine cervix to invasive cancers. On the other hand, HITS expression is preserved in aggressive types of cancers, such as scirrhous-type gastric and breast cancers, which are characterized by distinct genetic alterations and rapid growth or invasions $(48,49)$. Because forced expression of HITS inhibited cancer cell proliferation in response to growth factors and tumor xenograft growth (Fig. 5) (16), we assume that HITS expression influences the primary tumor growth per se during tumor development, but that it does not influence the invasion or metastasis, e.g., tumor spread such as scirrhous-type or lymphnode metastasis.

A clear boundary of HITS expression levels was apparent between cancer and non-cancerous lesions e.g. inflammation, benign tumor, and precancerous lesions in thyroid, cervical uterine and testicular tissues (Figs. 3 and 4). Diagnosis of malignancy in thyroid tumors is a difficult task performed using a combination of pathologic examination, blood tests including those of thyroid hormones, and image diagnostic methods such as computed tomography, ultrasound sonography, and scintigraphy. Fine needle aspiration cytology (FNAC) is a useful method to differentiate benign and malignant nodules. However, the sensitivity and specificity of FNAC has been reported, respectively, as $65-98 \%$ and $73-100 \%$ (50). Our statistical analysis revealed that HITS expression levels in both papillary and follicular types of thyroid carcinoma were significantly lower than in non-cancerous lesions such as adenoma, goiter, and thyroiditis (Fig. 3), suggesting the possibility of using immunohistochemical examination of HITS expression to complement the routine pathology diagnosis of thyroid cancer.

Persistent HPV infection is the primary cause of CIN and invasive cervical cancer. CIN encompasses a continuum of morphologic changes arising in the basal layer of the stratified squamous epithelium of the transformation zone. The mean age of women with CIN is about 15 years younger than the age of those with invasive cancer, suggesting a slow progression of CIN to invasive carcinoma (31). Our result that the HITS expression level was significantly different between CIN and invasive cervical cancer (Fig. 4) indicates that the HITS expression was lost during a course of CIN progression to invasive carcinoma following HPV infection.

The molecular mechanism underlying the biological functions of FAM107 remains unclear. The C-terminal variable regions of FAM107 with a coiled-coil domain are expected to be involved in regulating gene transcription. Recently, it was reported that a point mutation in the C-terminal region of HITS (chromosome10:14603968 $\mathrm{C} \bullet \mathrm{G} \rightarrow \mathrm{T} \bullet \mathrm{A}$ transition) is frequently observed in the primary tumor, brain metastasis and especially in xenograft in genomic analyses of basal-like breast cancer (51). The protein sequence of the C-terminal region is unique to HITS, suggesting its role in tumorigenicity through the transcriptional regulation of oncogenes and tumor-suppressor genes. In contrast, C-terminal coiled-coil domain of FAM107A was reported to associate with and organize the actin and microtubular cytoskeletons and that these associations are essential for focal adhesion (FA) disassembly and cell invasion (14). In fact, FAM107A is highly expressed in the invasive component of gliomas and drives invasion as a new cytoskeletal crosslinker that regulates FA dynamics and cell movement. As expected from the difference of C-terminal sequences between HITS and FAM107A, our preliminary experiments showed no association of HITS with the actin cytoskeleton or regulation of FA dynamics either in neural cells or cancer cells (unpublished observation). The function and molecular interaction of the N-terminal conserved domain (DUF1151) of FAM107 remain to be elucidated, but might play an important role in interacting with other proteins to transduce cellular signals and modulate gene transcription in common with FAM107 family proteins as candidate tumor suppressors.

In conclusion, our study clarified HITS as a potential tumor suppressor belonging to HSPs and as a useful marker for pathological diagnosis for use in cases since expression is often lost in carcinomas in various tissues in histopathologicaltype-specific manner, and involved in suppression of tumor growth.

\section{Acknowledgements}

We thank Atsuko Asaka, Kazumi Tanaka and Yumiko Hoshiba for their technical assistance. This study was supported by Grants-in-Aid for Scientific Research from the Japanese Ministry of Education, Culture, Sports, Science and Technology; Collaborative Research (C2011-1) of Kanazawa Medical University. 


\section{References}

1. Rual JF, Venkatesan K, Hao T, et al: Towards a proteome-scale map of the human protein-protein interaction network. Nature 437: 1173-1178, 2005.

2. Ewing RM, Chu P, Elisma F, et al: Large-scale mapping of human protein-protein interactions by mass spectrometry. Mol Syst Biol 3: 89, 2007.

3. Stelzl U, Worm U, Lalowski M, et al: A human protein-protein interaction network: a resource for annotating the proteome. Cell 122: 957-968, 2005

4. Wang YL, Faiola F, Xu M, Pan S and Martinez E: Human ATAC Is a GCN5/PCAF-containing acetylase complex with a novel NC2-like histone fold module that interacts with the TATA-binding protein. J Biol Chem 283: 33808-33815, 2008.

5. Frijters R, Fleuren W, Toonen EJ, et al: Prednisolone-induced differential gene expression in mouse liver carrying wild type or a dimerization-defective glucocorticoid receptor. BMC Genomics 11: 359, 2010.

6. Yamato T, Orikasa K, Fukushige S, Orikasa S and Horii A: Isolation and characterization of the novel gene, TU3A, in a commonly deleted region on 3 p14.3-->p14.2 in renal cell carcinoma. Cytogenet Cell Genet 87: 291-295, 1999.

7. Wang L, Darling J, Zhang JS, et al: Loss of expression of the DRR 1 gene at chromosomal segment 3 p21.1 in renal cell carcinoma. Genes Chromosomes Cancer 27: 1-10, 2000.

8. van den Boom J, Wolter M, Blaschke B, Knobbe CB and Reifenberger G: Identification of novel genes associated with astrocytoma progression using suppression subtractive hybridization and real-time reverse transcription-polymerase chain reaction. Int J Cancer 119: 2330-2338, 2006.

9. Vanaja DK, Ballman KV, Morlan BW, et al: PDLIM4 repression by hypermethylation as a potential biomarker for prostate cancer. Clin Cancer Res 12: 1128-1136, 2006.

10. Liu Q, Zhao XY, Bai RZ, et al: Induction of tumor inhibition and apoptosis by a candidate tumor suppressor gene DRR1 on 3p21.1. Oncol Rep 22: 1069-1075, 2009.

11. Kholodnyuk ID, Kozireva S, Kost-Alimova M, Kashuba V Klein $G$ and Imreh $S$ : Down regulation of $3 p$ genes, LTF, SLC38A3 and DRR1, upon growth of human chromosome 3-mouse fibrosarcoma hybrids in severe combined immunodeficiency mice. Int J Cancer 119: 99-107, 2006.

12. Zhao XY, Liang SF, Yao SH, et al: Identification and preliminary function study of Xenopus laevis DRR1 gene. Biochem Biophys Res Commun 361: 74-78, 2007.

13. Asano Y, Kishida S, Mu P, Sakamoto K, Murohara T and Kadomatsu K: DRR1 is expressed in the developing nervous system and downregulated during neuroblastoma carcinogenesis. Biochem Biophys Res Commun 394: 829-835, 2010.

14. Le PU, Angers-Loustau A, de Oliveira RMW, et al: DRR drives brain cancer invasion by regulating cytoskeletal-focal adhesion dynamics. Oncogene 29: 4636-4647, 2010.

15. Schmidt MV, Schulke JP, Liebl C, et al: Tumor suppressor down-regulated in renal cell carcinoma 1 (DRR1) is a stressinduced actin bundling factor that modulates synaptic efficacy and cognition. Proc Natl Acad Sci USA 108: 17213-17218, 2011.

16. Nakajima H, Ishigaki Y, Xia QS, et al: Induction of HITS, a newly identified family with sequence similarity 107 protein (FAM107B), in cancer cells by heat shock stimulation. Int J Oncol 37: 583-593, 2010.

17. Jameel A, Skilton RA, Campbell TA, Chander SK, Coombes RC and Luqmani YA: Clinical and biological significance of HSP89 alpha in human breast cancer. Int J Cancer 50: 409-415, 1992.

18. Takayama S, Reed JC and Homma S: Heat-shock proteins as regulators of apoptosis. Oncogene 22: 9041-9047, 2003.

19. Foster CS, Dodson AR, Ambroisine L, et al: Hsp-27 expression at diagnosis predicts poor clinical outcome in prostate cancer independent of ETS-gene rearrangement. Br J Cancer 101 : 1137-1144, 2009.

20. Ciocca DR, Clark GM, Tandon AK, Fuqua SA, Welch WJ and McGuire WL: Heat shock protein hsp70 in patients with axillary lymph node-negative breast cancer: prognostic implications. J Natl Cancer Inst 85: 570-574, 1993.

21. Nanbu K, Konishi I, Mandai M, et al: Prognostic significance of heat shock proteins HSP70 and HSP90 in endometrial carcinomas. Cancer Detect Prev 22: 549-555, 1998.

22. Jolly C and Morimoto RI: Role of the heat shock response and molecular chaperones in oncogenesis and cell death. J Nat Cancer Inst 92: 1564-1572, 2000.
23. Rohde M, Daugaard M, Jensen MH, Helin K, Nylandsted J and Jaattela M: Members of the heat-shock protein 70 family promote cancer cell growth by distinct mechanisms. Genes Dev 19: 570-582, 2005

24. Whitesell L and Lindquist SL: HSP90 and the chaperoning of cancer. Nat Rev Cancer 5: 761-772, 2005.

25. Mai W, Kawakami K, Shakoori A, et al: Deregulated GSK3(beta) sustains gastrointestinal cancer cells survival by modulating human telomerase reverse transcriptase and telomerase. Clin Cancer Res 15: 6810-6819, 2009.

26. Belkacemi Y, Bousquet G, Marsiglia H, et al: Phyllodes tumor of the breast. Int J Radiat Oncol Biol Phys 70: 492-500, 2008.

27. Woodward WA, Strom EA, Tucker SL, et al: Changes in the 2003 American Joint Committee on Cancer staging for breast cancer dramatically affect stage-specific survival. J Clin Oncol 21: 3244-3248, 2003.

28. Pusztai L, Cristofanilli $M$ and Paik S: New generation of molecular prognostic and predictive tests for breast cancer. Semin Oncol 34: S10-S16, 2007.

29. Kollias J, Elston CW, Ellis IO, Robertson JF and Blamey RW: Early-onset breast cancer - histopathological and prognostic considerations. Br J Cancer 75: 1318-1323, 1997.

30. Bosch FX and de Sanjose S: Chapter 1: Human papillomavirus and cervical cancer - burden and assessment of causality. J Natl Cancer Inst Monogr pp3-13, 2003.

31. Kivlahan $\mathrm{C}$ and Ingram E: Papanicolaou smears without endocervical cells. Are they inadequate? Acta Cytol 30: 258-260, 1986.

32. Awakura Y, Nakamura E, Ito N, Kamoto T and Ogawa O: Methylation-associated silencing of TU3A in human cancers. Int J Oncol 33: 893-899, 2008.

33. Park K, Han S, Kim HJ, Kim J and Shin E: HER2 status in pure ductal carcinoma in situ and in the intraductal and invasive components of invasive ductal carcinoma determined by fluorescence in situ hybridization and immunohistochemistry. Histopathology 48: 702-707, 2006.

34. Dai C, Whitesell L, Rogers AB and Lindquist S: Heat shock factor 1 is a powerful multifaceted modifier of carcinogenesis. Cell 130: 1005-1018, 2007.

35. Jego G, Hazoume A, Seigneuric R and Garrido C: Targeting heat shock proteins in cancer. Cancer Lett: Nov 13, 2010 (available online)

36. Wang RE: Targeting heat shock proteins $70 / 90$ and proteasome for cancer therapy. Curr Med Chem 18: 4250-4264, 2011.

37. Khalil AA, Kabapy NF, Deraz SF and Smith C: Heat shock proteins in oncology: diagnostic biomarkers or therapeutic targets? Biochim Biophys Acta 1816: 89-104, 2011.

38. Dalton LW, Page DL and Dupont WD: Histologic grading of breast carcinoma. A reproducibility study. Cancer 73: 2765-2770, 1994.

39. Tsuda H, Akiyama F, Kurosumi M, Sakamoto G and Watanabe T: Establishment of histological criteria for high-risk node-negative breast carcinoma for a multi-institutional randomized clinical trial of adjuvant therapy. Japan National Surgical Adjuvant Study of Breast Cancer (NSAS-BC) Pathology Section. Jpn J Clin Oncol 28: 486-491, 1998.

40. Mylonas I, Makovitzky J, Jeschke U, Briese V, Friese K and Gerber B: Expression of Her2/neu, steroid receptors (ER and PR), Ki67 and p53 in invasive mammary ductal carcinoma associated with ductal carcinoma in situ (DCIS) versus invasive breast cancer alone. Anticancer Res 25: 1719-1723, 2005.

41. Lower EE, Glass EL, Bradley DA, Blau R and Heffelfinger S: Impact of metastatic estrogen receptor and progesterone receptor status on survival. Breast Cancer Res Treat 90: 65-70, 2005.

42. Wolff AC, Hammond ME, Schwartz JN, et al: American Society of Clinical Oncology/College of American Pathologists guideline recommendations for human epidermal growth factor receptor 2 testing in breast cancer. J Clin Oncol 25: 118-145, 2007.

43. Narod SA, Brunet JS, Ghadirian P et al: Tamoxifen and risk of contralateral breast cancer in BRCA1 and BRCA 2 mutation carriers: a case-control study. Hereditary Breast Cancer Clinical Study Group. Lancet 356: 1876-1881, 2000.

44. Schnitt SJ: Estrogen receptor testing of breast cancer in current clinical practice: what's the question? J Clin Oncol 24: 1797-1799, 2006.

45. Farabegoli F, Champeme MH, Bieche I, et al: Genetic pathways in the evolution of breast ductal carcinoma in situ. J Pathol 196: 280-286, 2002 .

46. Jo BH and Chun YK: Heterogeneity of invasive ductal carcinoma: proposal for a hypothetical classification. J Korean Med Sci 21: 460-468, 2006. 
47. Steinman S, Wang J, Bourne P, Yang Q and Tang P: Expression of cytokeratin markers, ER-alpha, PR, HER-2/neu, and EGFR in pure ductal carcinoma in situ (DCIS) and DCIS with co-existing invasive ductal carcinoma (IDC) of the breast. Ann Clin Lab Sci 37: 127-134, 2007.

48. Lauren PA and Nevalainen TJ: Epidemiology of intestinal and diffuse types of gastric carcinoma. A time-trend study in Finland with comparison between studies from high- and low-risk areas. Cancer 71: 2926-2933, 1993.
49. Walker RA: The complexities of breast cancer desmoplasia. Breast Cancer Res 3: 143-145, 2001.

50. Haberal AN, Toru S, Ozen O, Arat Z and Bilezikci B: Diagnostic pitfalls in the evaluation of fine needle aspiration cytology of the thyroid: correlation with histopathology in 260 cases. Cytopathology 20: 103-108, 2009.

51. Ding L, Ellis MJ, Li S, et al: Genome remodelling in a basal-like breast cancer metastasis and xenograft. Nature 464: 999-1005, 2010. 